\section{Automated TEM Sample Preparation}

Wayne D. Kaplan (Technion-Israel Institute of Technology), Efrat Raz \& Colin Smith (SELA USA, Inc.)

\section{Abstract}

The rising demand in the semiconductor industry for higher spatial resolution in the analysis of device defects has focused attention on the use of transmission electron microscopy (TEM). However, conventional TEM sample preparation can be difficult and time-consuming, and, depending on the operator, may result in a low yield of quality specimens. One solution to this problem is the use of focused ion beam (FIB) milling for the final stage of TEM sample preparation. However, specimens have to be mechanically thinned prior to FIB and the need to characterize specific devices requires a pre-FIB preparation method that can target specific features on the wafer. We will discuss an innovative and automated solution that isolates specific devices and prepares TEM specimens for subsequent FIB thinning. The complete pre-FIB preparation takes less than 30 minutes and yields a sample in which the targeted feature is positioned a specific distance from the sample edge, thereby minimizing final FIB milling time. The output specimen is automatically packaged for FIB milling and TEM analysis. We also present drawings of the process flow and examples showing TEM results from tungsten filled vias.

\section{Introduction}

Transmission electron microscopy (TEM) is a fundamental characterization technique in materials science, and one of the only methods that provides crystallographic, morphological and chemical information with high spatial resolution ${ }^{1}$. While TEM is extensively used in basic research and development, it is rarely used in industry for process monitoring, due to the length of time and operator skill required to prepare TEM specimens. With the continuing reduction in semiconductor device sizes process monitoring increasingly requires TEM investigations to provide microstructural characterization not available from scanning electron microscopy (SEM). The demanding specifications for quality TEM specimen preparation are currently the primary limitation preventing increased use of TEM in the semiconductor industry.

The requirements for a quality TEM specimen are extremely rigid. Specimens are normally $3.0 \mathrm{~mm}$ in diameter, and often less than $100 \mu \mathrm{m}$ in thickness. The center region of the specimen must be further thinned to electron transparency (less than $1 \mu \mathrm{m}$ ) and must include the region of interest. While the preparation of planar-view specimens from bulk materials is fairly straightforward, the preparation of cross section specimens to investigate thin films and devices on a substrate is extremely complicated.

Cross sections of thin films are usually prepared by first sectioning the substrate, and gluing sections 'face-to-face', creating a 3.0 $\mathrm{mm}$ thick slab of material containing the thin films at its center. For substrates with a thickness of less than 1.5 f $\mathrm{mm}$, additional sections are glued 'face-to-back' in order to reach a total slab thickness of $3.0 \mathrm{~mm}$. To provide a final specimen with sufficient mechanical rigidity, the sectioned slabs are then inserted and glued inside a metal or ceramic tube $e^{2-3}$. Disks of $3.0 \mathrm{~mm}$ di- $\vec{\circ}$ ameter are then sectioned from the tube and mechanically thinned to less than $100 \mu \mathrm{m}$ in thickness. Chemical or electro-chemicalư thinning techniques are usually not possible for cross-section $\overrightarrow{0}$ specimens, due to the varying thinning rates of the different mate- ${ }^{\circ}$ rials, so broad-beam ion-milling is the choice for thinning the center of the disk to electron transparency. In order to induce prefer- $\vec{\infty}$ ential ion-milling at the center of the specimen (the region containing the thin films) mechanical dimpling of the center region is nor $-\frac{\bar{v}}{\sigma}$ mally required prior to ion-milling.

While this method can produce quality cross-section speci-⿳亠二口犬. mens, the process is extremely time consuming, and requirese great individual expertise. In addition, the process yield is unac- $-\frac{\Omega}{3}$ ceptably low with only a fraction of completed specimens suffi-⿳亠丷厂 ciently thin for TEM investigations. Finally, the method is not viable for TEM investigations of specific devices and defects since it is not possible to ensure that the region of interest will be located in $\frac{0}{n}$. the electron transparent region at the center of the specimen.

In recent years focused ion beam (FIB) milling has been used to produce cross section TEM specimens of individual devices ${ }^{47}$.

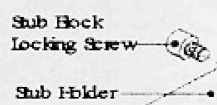

1

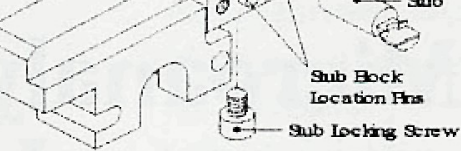

Inse rt Ne w Stub

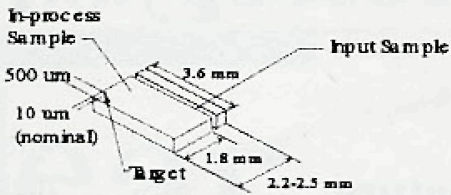

After Pre limina ry Sa wing

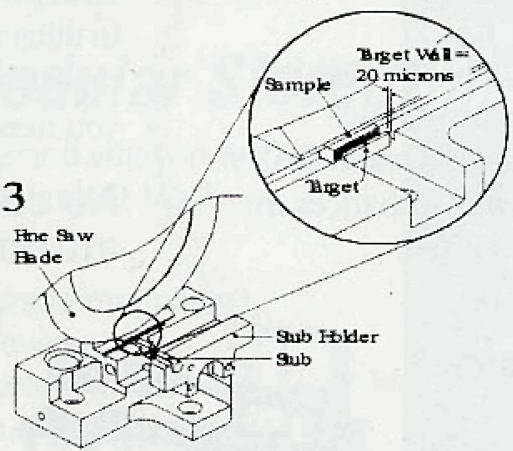

After First Sa wing

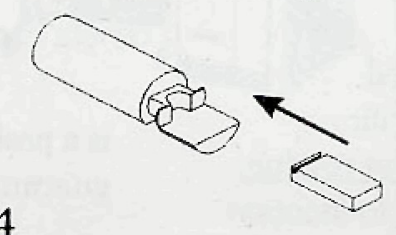

Ghing Sample to Stub
5

After Sec ond Sa wing

6

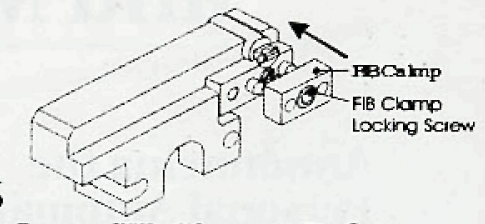

Inseit FBClamp, Before Third Sa wing

7

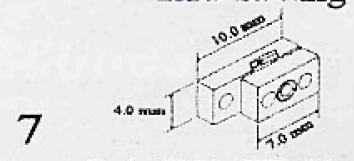

Stub Wh FIBClamp
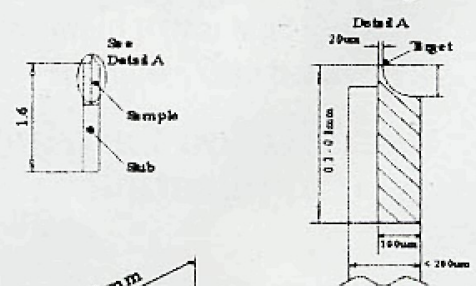

8

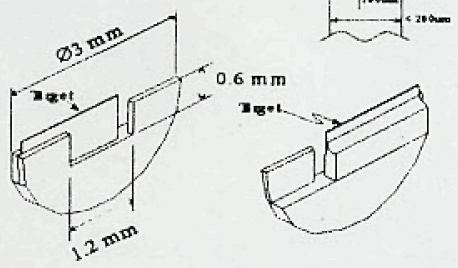

Output Sample Dimensions

Figure 1: The pre-FIB sample preparation process flow. 


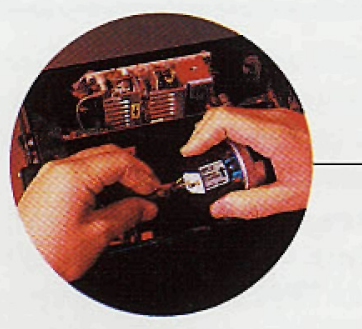

Pre-Aligned, 2000 Hour Lamp

Proprietary 50W metal halide lamp produces a richer spectral profile than conventional $100 \mathrm{~W}$ mercury arc lamps for additional fluorophore excitation. Pre-aligned lamp is simple to change and has extra-long life.

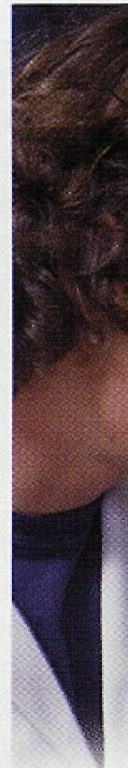

\section{Intelli-Lamp ${ }^{\text {TM }}$ System}

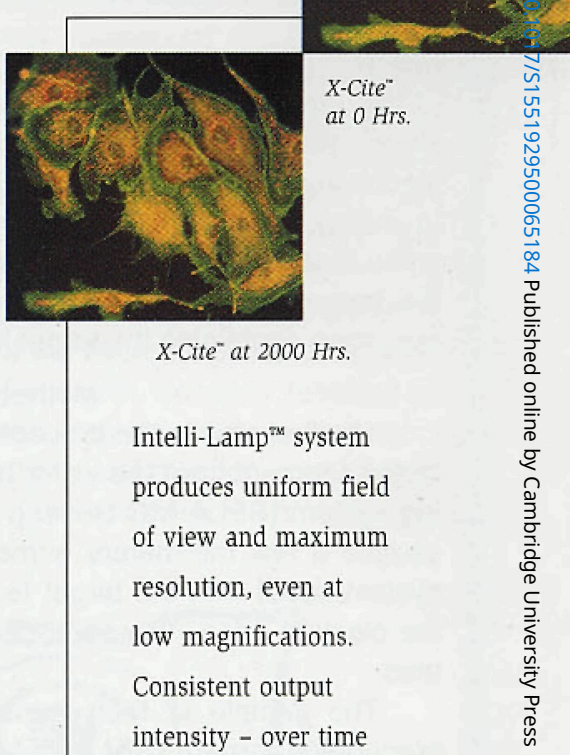

\section{Introducing the EFOS X-Cite}

\section{Changing the world of fluorescence illumination.}

\section{FOCUS YOUR ENEREY WHERE IT COUNTS - ON YOUR WORK}

\section{Productivity by Design}

Designed for easier use and better results. Snapon connector makes set-up simple - and you only have to do it once. Remote shutter eliminates vibration. Liquid light guide eliminates sample heating.

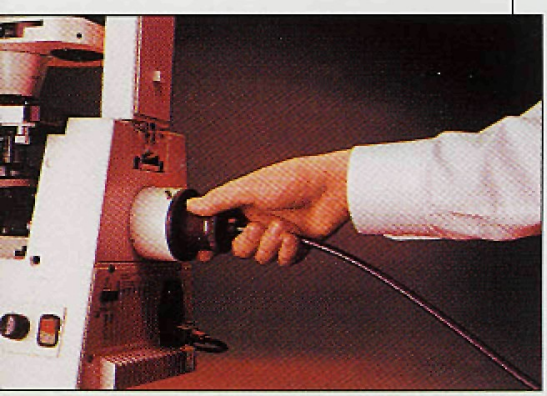

\section{당 $0 \longdiv { x \text { -cite } }$ (a) 10.2 (K) \.
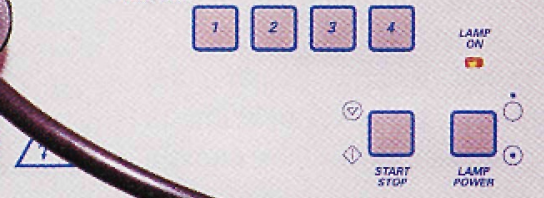

Unique Foot Pedal Control

Leaves hands free for navigation and micromanipulation. Reduces photobleaching. the revolutionary new $\mathrm{X}$-Cite ${ }^{\mathrm{Tx}}$ gives you levels of convenience and control never before available in a fluorescence illuminator. It's easier to use, less expensive to operate and meets international safety and EMC standards for laboratory equipment. Take a closer look at how the $\mathrm{X}$-Cite ${ }^{\text {Th }}$ can help you work faster, safer and smarter:

Visit www.efos.com/focus or call 1-800-668-8752.

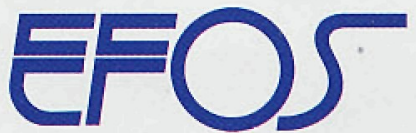

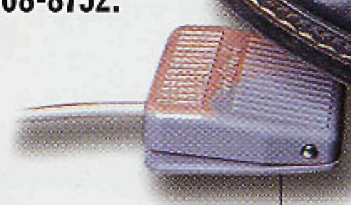




\section{Automated TEM Sample Preparation}

Continued from page 14

FIB milling has the advantage of permitting constant monitoring of the target using the secondary electron image. However, since FIB is inherently slow, most FIB preparations require the mechanical thinning of the specimen prior to milling. Also prior to milling, the wafer must be sectioned in a manner that ensures that the region of interest can be thinned by FIB. Using the process described below, we can prepare a $20 \mu \mathrm{m}$ thin specimen containing the target in less than 30 minutes.

\section{Methodology}

The first step in the process is the precision cleaving of the target segment from the wafer using an automated Microcleaving system (SELA MC Series). This produces a pre-FIB input sample a few millimeters across (exact size depends on the system used) with the target feature at the chosen offset from the cleaved edge. The Microcleaving process takes 7-20 minutes.

The sample is then transferred to the pre-FIB sample preparation system (SELA TEMstation) where processing proceeds as follows (step numbers refer to the process flow diagram shown in figure 1):

1) The operator loads consumables, a filled glue capsule and two needles, into the stack and then inserts a new stub into the stub holder. Then the operator places the input sample on the pre-sawing chuck.

2) The system automatically performs a preliminary sawing operation to create a specifically dimensioned in-process sample if required. The cleaved edge and nearby area of interest are not disturbed by this or any subsequent sawing operation.

3) The system automatically transfers the in-process sample from the pre-sawing chuck to the main chuck and performs an automatic alignment procedure to ensure that the cleaved sample edge is properly aligned with the saw blade prior to the first sawing operation. Using a very fine blade, the system then cuts a channel parallel to the cleaved edge, creating a $20 \mathrm{~mm}$ (nominal) thin wall containing the target. After completing the sawing operation, the system monitor displays an optical image of the thinned wall, allowing the operator to confirm sample quality before proceeding.

4) After the first sawing, the system automatically bonds the specimen to the stub and aligns the specimen for the second sawing.

5) The second sawing trims away the bulk of the sample and the underlying stub, leaving the $20 \mu \mathrm{m}$ thin wall and the area of interest untouched and bonded to the stub.

6) The operator now removes the stub holder with the stub and sample in place and applies the FIB clamp to the portion of the stub that will become the TEM sample carrier.

7) After the operator returns the stub assembly, the system performs a final sawing operation to separate the sample, TEM sample carrier, and FIB clamp from the remainder of the stub. The operator may now transfer the sample, grid and clamp directly to a FIB workstation for final thinning.

8) After thinning in the FIB system and removing the clamp, the sample and grid are ready for direct insertion into a TEM sample holder.

The finished pre-FIB specimen contains one cleaved edge (a high quality edge) and one sawed edge. Detailed SEM in- vestigations have shown no significant micro-cracking (Such surface flaws could act as crack initiation sites and lead to failure of the specimen during handling.) due to cleaving or sawing, and a maximum of $2 \mu \mathrm{m}$ chipping on the sawed edge.

The entire pre-FIB process takes approximately 30 minutes. The resulting $20 \mu \mathrm{m}$ thin specimen typically requires 1 to 1.5 hours of FIB milling time to produce the final TEM sample. The system is fully automated, and does not require operator intervention from the time the target is designated until the FIB clamp is applied. It is well suited to a multi-user environment, and requires no expertise or previous experience. In our laboratory we routinely achieve throughput of 3-4 samples per hour. The finished sample requires no cleaning and is never exposed to water during the process.

\section{Examples}

Figures 2 through 7 show various stages in the preparation of a TEM sample using the automated system described above. The targeted structures are tungsten vias in an integrated circuit fabricated on a conventional silicon wafer.

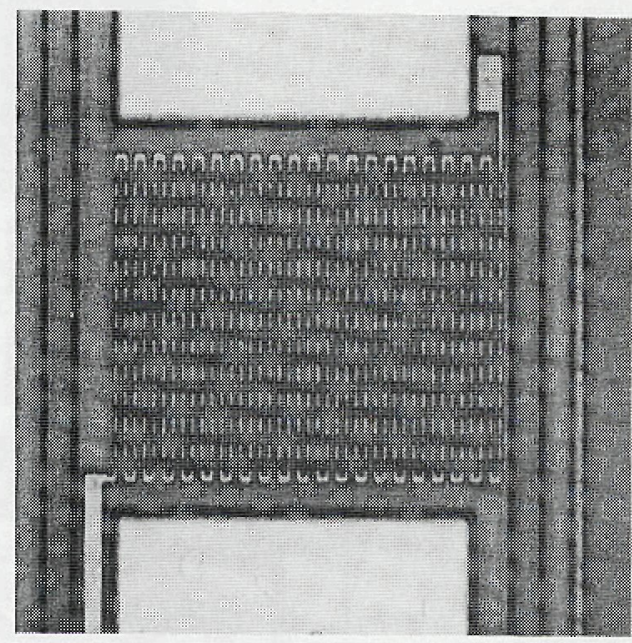

Figure 2: The process begins with isolation of the region of interest using the integrated optical microscope and video display of the microcleaving system. These optical micrographs show the region of interest, from which the TEM cross-section will be prepared.

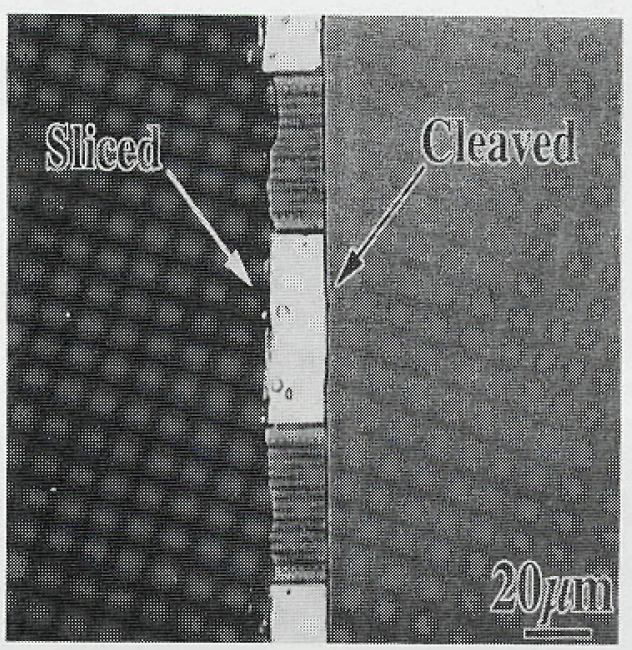

Figure 3: After the operator designates the target, the system slices a channel (dark region on left) parallel to the cleaved sample edge leaving a $20 \mu \mathrm{m}$ thin wall containing the target.

Continued on page 18 

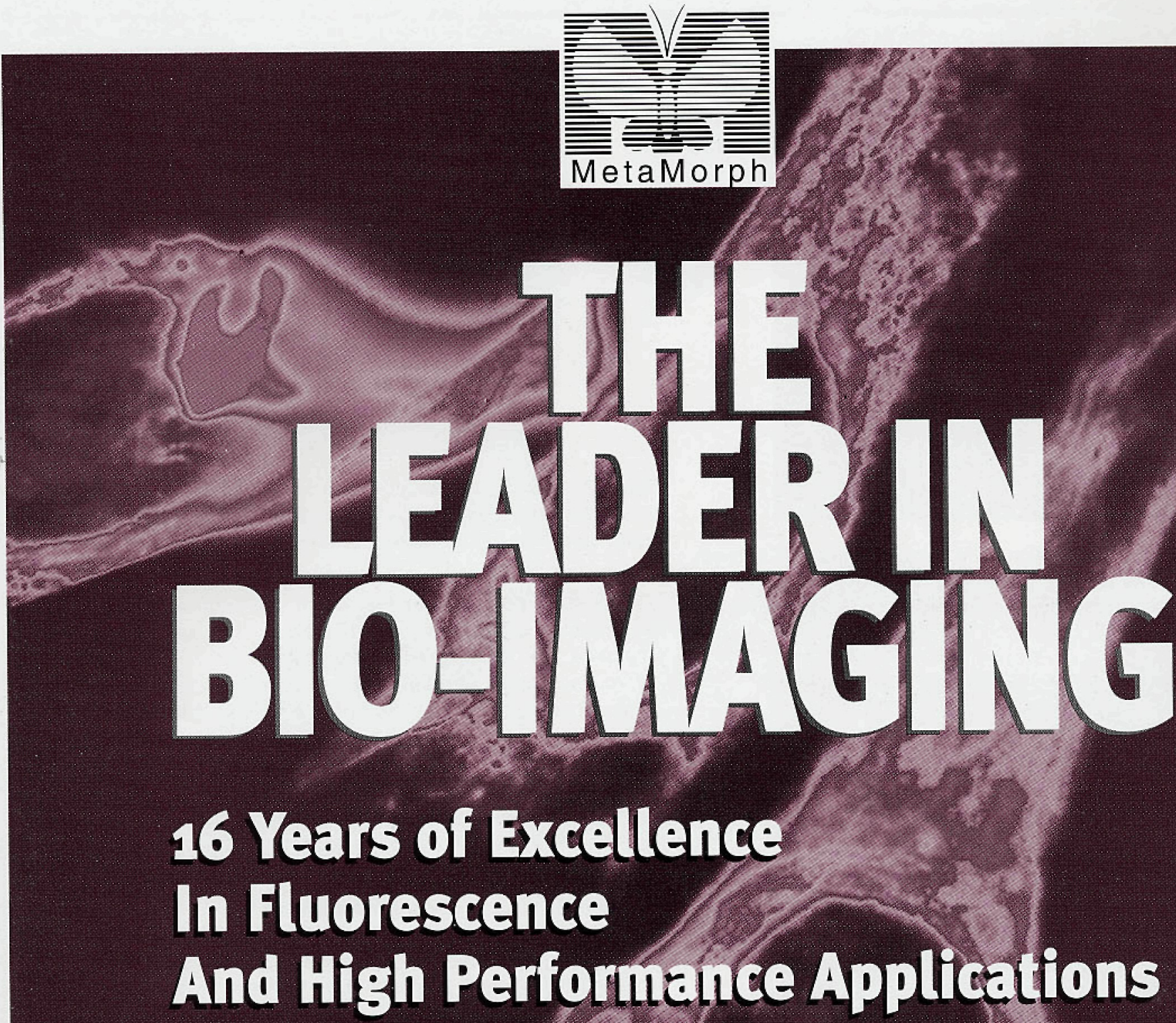

6-Dimensional Imacing GFP

FLUORESCENCE

IMMUNOCYTOCHEMISTRY

Intracellular Calcium

LIVE/DEAD ASSAYS
Motion AnaLysis

\& Particle trackine

High Speed Ratio

FLUORESCENCE

HIGH SREED Z-SERILS

TIME LAPSE

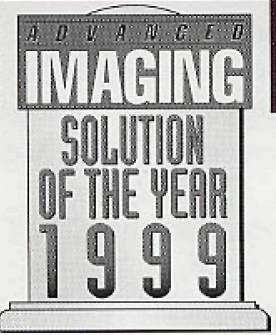

sales@image1.com
UNIVERSAL IMAGING CORPORATION

www.image1.com 
Automated TEM Sample Preparation

Continued from page 16

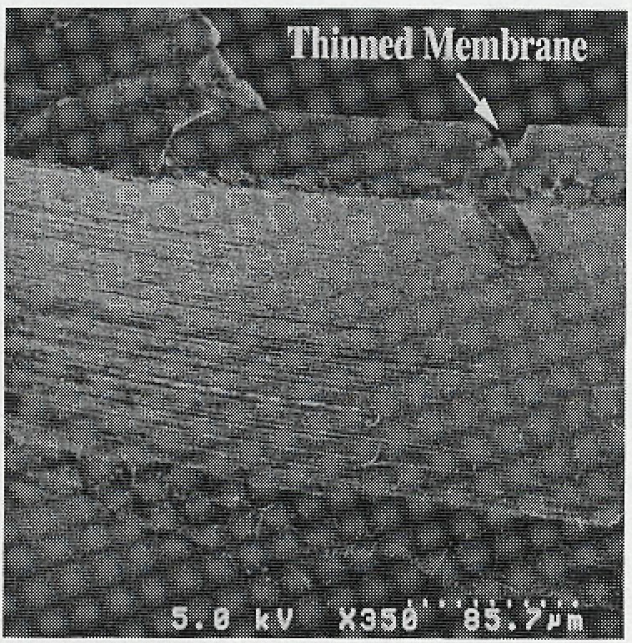

Figure 4: A low magnification SEM micrograph of a TEM-ready specimen shows the $20 \mu \mathrm{m}$ thin wall containing the target and the FIB milled pits on either side of the final thin membrane.

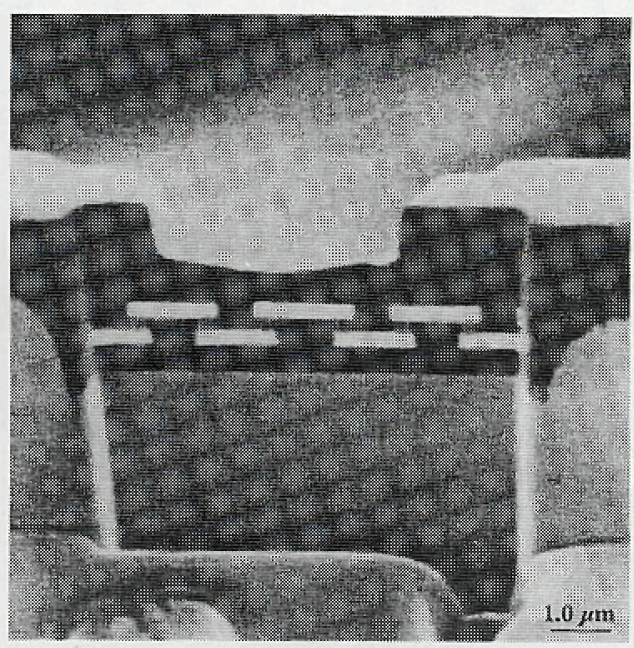

Figure 5: This higher magnification SEM micrograph shows the final thin membrane containing the region of interest (after FIB milling).

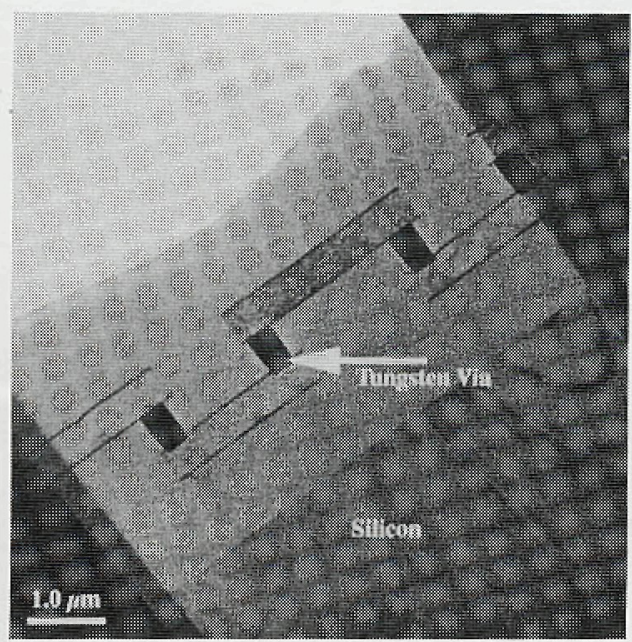

Figure 6: This low magnification bright field TEM micrograph shows the region of interest.

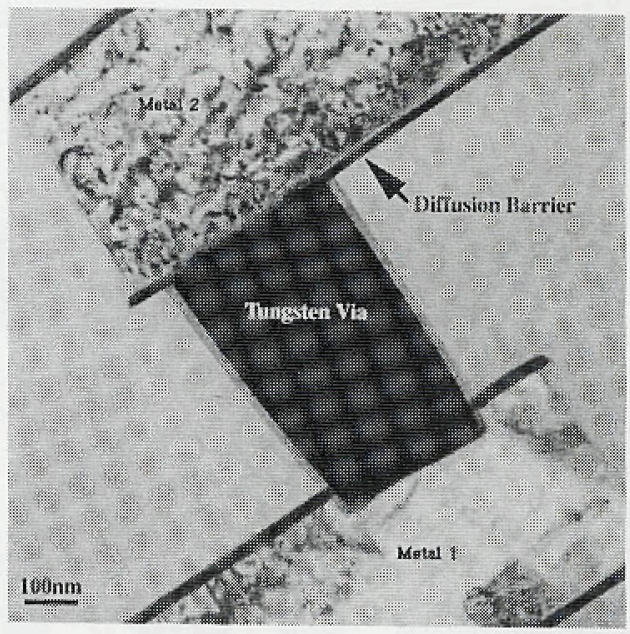

Figure 7: This higher magnification bright field TEM micrograph? shows the morphology of the via, the metal lines, and the TiN diffusion barrier located between the metal and the via.

\section{Summary \& Conclusions}

We have described an innovative and automated technique for preparing cross sectional TEM samples of selected device structures. The entire pre-FIB preparation, including Microcleaving, routinely takes about 30 minutes. An important added benefit is the capability to position the target a specific distance from the sample edge, thus minimizing subsequent FIB milling time as well. The pre-FIB specimen is already packaged for FIB milling and TEM examination. All of these contribute to the significant time savings we achieve in our operations from automated TEM sample preparation. And more so, perhaps, than in any other industry, in semiconductor manufacturing, time is money.

\section{Acknowledgements}

The authors wish to thank Ron Oviedo and Kim Kisslinger of National Semiconductor Corporation in South Portland, Maine for providing the sample preparation and images in this paper and their input to this article.

\section{References}

1. D. B. Williams and C. B. Carter, Transmission Electron Microscopy, Plenum Press, N.Y. (1996).

2. A. Strecker, U. Salzberger, and J. Mayer, "Specimen Preparation for Transmission Electron Microscopy; Reliable Method for Cross-Sections and Brittle Materials," PraktischeMetallographie, 30[10]:482-495 (1993).

3. D. Brandon and W.D. Kaplan, Microstructural Characterization of Materials, John Wiley \& Sons, N.Y. (1999).

4. J.F. Walker, D.F. Moore, and J.T. Whitney, "Focused Ion Beam Processing for Microscale Fabrication," MicroelectronicEngineering, 30[1-4]:517-522 (1996).

5. Y. Kitano, Y. Fujikawa, T. Kamino, T. Yaguchi, and H. Saka, "TEM Observation of Micrometer-Sized Ni Powder Particles Thinned by FIB Cutting Technique," J. Electron Microscopy, 44 [5]:410-413 (1995).

6. Y. Kitano, Y. Fujikawa, H. Takeshita, T. Kamino, T. Yaguchi, $\mathrm{H}$. Matsumoto, and $\mathrm{H}$. Koike, "TEM Observation of Mechanically Alloyed Powder Particles (MAP of Mg-Zn Alloy Thinned by the FIB Cutting Technique," J. Electron Microscopy, 44[5]:376383 (1995).

7. M.H.F. Overwijk, F.C. Van Den Heuvel, and C.W.T. Bulle-Lieuwma, "Novel Scheme for the Preparation of Transmission Electron Microscopy Specimens with a Focused lon Beam," J. Vacuum Science \& Tech., 11[6]:2021-2024 (1993). 
Digital technology is changing the way we look at the world.

Upgrade your existing TEM to obtain high resolution digital images and digital movies. DualView adds a new dimension for the material science or bioscience field. Isn't it time you made the move to digital ?

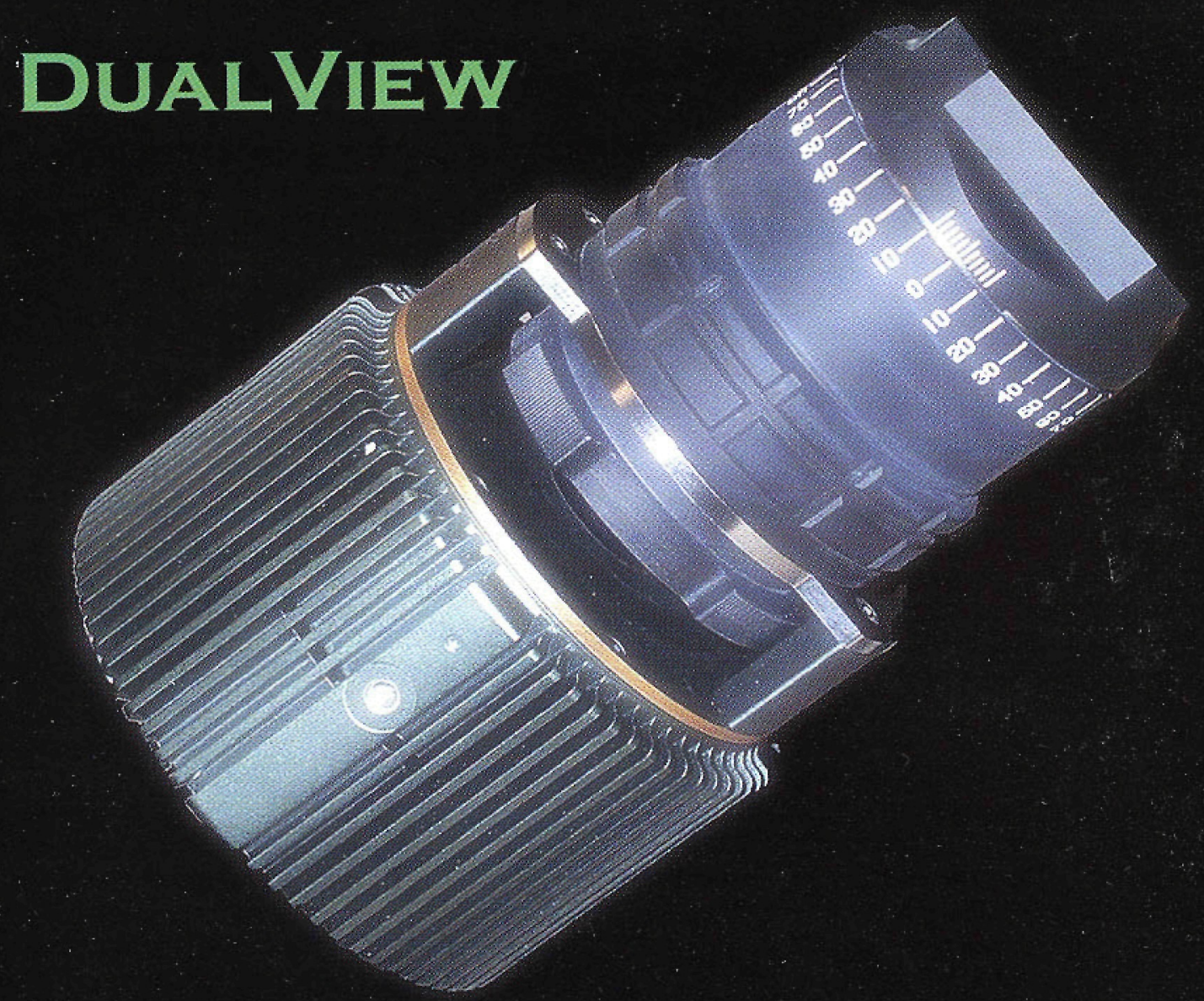

\section{are

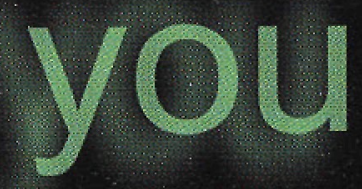

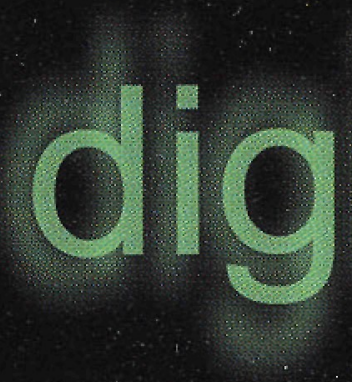
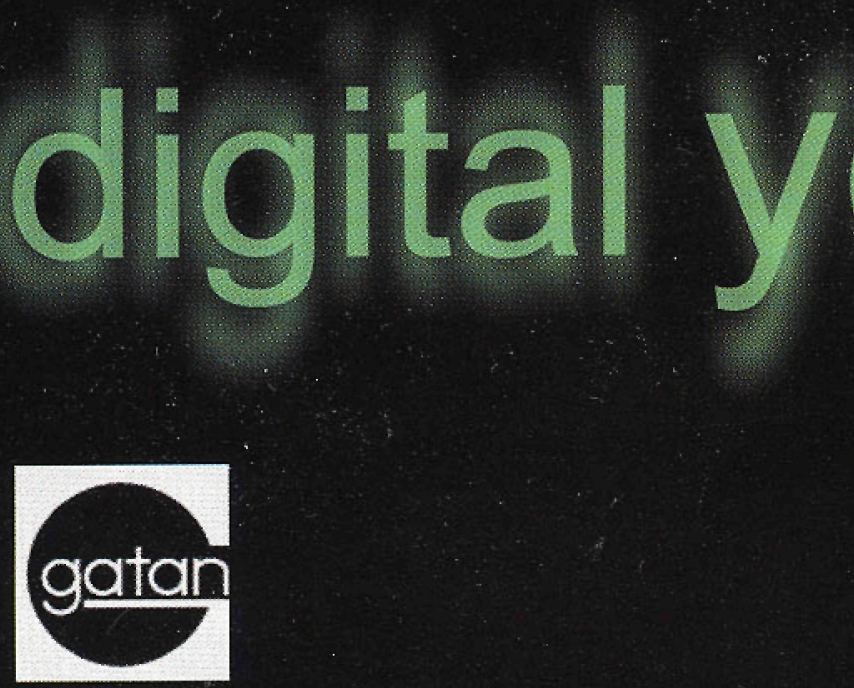

Digital Integrity 